Postoperative Akutschmerztherapie

\title{
Es besteht noch Optimierungsbedarf
}

— In der Akutschmerzbehandlung - insbesondere nach kleineren und deshalb häufig unterschätzten Eingriffen - bestehe noch deutlicher Verbesserungsbedarf, erläuterte Prof. Dr. Wolfgang Koppert, Medizinischen Hochschule Hannover, bei einem Hintergrundgespräch. Etwa ein Viertel der Patienten leidet am ersten postoperativen Tag unter starken, ungefähr die Hälfte unter mäßig starken bis starken Schmerzen. Unter Belastung sind postoperative Schmerzen bei vielen Patienten in den ersten 24 Stunden unakzeptabel hoch, wie auch das Forschungsprojekt "Schmerzfreies Krankenhaus" gezeigt hat.

Neben der Bereitstellung effektiver Akutschmerzmittel wie Tapentadol (Palexia ${ }^{\circledR}$ IR) möchte Grünenthal mit der Initiative Change Pain ${ }^{\circledast}$ die Versorgung von Schmerzpatienten verbessern, die Schmerzchronifizierungsrate reduzieren und eine mechanismen-orientierte Schmerztherapie als therapeutisches Basisschema etablieren. Seit 2015 werden die Inhalte und Services von
Change Pain ${ }^{\circledast}$ um den Bereich des Akutschmerzes mit besonderem Fokus auf postoperativen Schmerzen erweitert (www. change-pain.de).

Um die Akutschmerzkontrolle zu verbessern, ist laut Koppert eine interdisziplinäre Kooperation sowie die aktive Einbeziehung des Patienten in die Therapieentscheidung erforderlich. Die Effekte einer qualifizierten Akutschmerztherapie zeigen sich in

_ Schnellerer Mobilisierung

_ Verbesserung der pulmonalen Funktion

_ Verringerung der gastrointestinalen Atoniedauer

_ Vermeidung von Chronifizierungen

- Zufriedenen Patienten und Personal

_ Gesteigerter Wettbewerbsfähigkeit der Klinik

„Die Versorgung des Patienten sollte daher so früh wie möglich erfolgen und an den individuellen Schmerzen des Patienten ausgerichtet sein", so Koppert. Optimal und in Leitlinien empfohlen sei eine nicht-invasive Analgetikagabe zum frühest möglichen

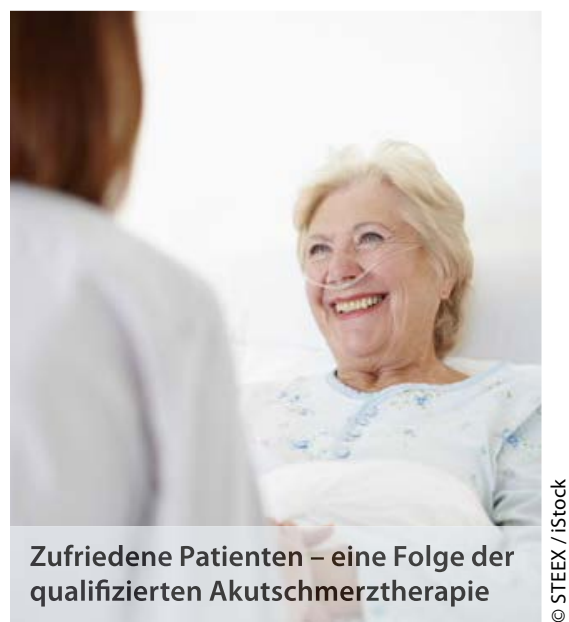

Zeitpunkt. Voraussetzung für eine nachhaltige Akutschmerztherapie ist zudem die sorgfältige Dokumentation der Anamnese und die Beurteilung der Schmerzstärke anhand der subjektiven Patienteneinschätzung, z.B. mit der numerischen Ratingskala (NRS).

(djb)

Hintergrundgespräch „Wertschätzung des postoperativen Schmerzmanagements (POMP) in der Klinik"; Frankfurt, 9. Juni 2015 (Veranstalter: Grünenthal GmbH)

\section{Aufklärungsinitiative \\ „Diabetes! Hören Sie auf Ihre Füße?"}

— Für viele Betroffene ist die diabetische Neuropathie eine große Unbekannte. Dies zeigen die wissenschaftlich evaluierten Ergebnisse von rund 1.000 podologischen Untersuchungen, die im Rahmen der Aufklärungsinitiative „Diabetes! Hören Sie auf Ihre Füße?" gewonnen wurden. Die bundesweite Initiative, die mit Informationen, Tipps und Aktionen zur Fußgesundheit allen Interessierten die Bedeutung von Früherkennung und Prävention der diabetischen Neuropathie näherbringen möchte, ist eine Gemeinschaftsaktion von Wörwag Pharma in Zusammenarbeit mit der Deutschen Diabetes-Stiftung (DDS) und Experten der Fachgebiete Diabetologie und Neurologie.

Die Tour steuerte zwischen Mai 2013 und Oktober 2014 Gesundheits- und Dia- betesmessen sowie Einkaufszentren in 26 deutschen Städten an, berichtete Prof. Dr. Oliver Schnell, Geschäftsführender Vorstand der Forschergruppe Diabetes e.V. am Helmholtz-Zentrum München und Kurator der DDS. Dabei wurde interessierten Bürgern auch ein kostenloser FußCheck durch einen Podologen angeboten, der Temperatur-, Druck- und Vibrationswahrnehmung sowie die Palpation der Fußpulse umfasste. Die Auswertung von 983 Fuß-Checks ergab bei gut der Hälfte einen Verdacht auf das Vorliegen einer Neuropathie. „Bei mehr als der Hälfte der Untersuchten mit bekanntem Typ-2-Diabetes wurden Hinweise für eine beginnende oder klinisch manifeste Neuropathie festgestellt. Sogar jeder Vierte ohne bekannten Diabetes wies Anzeichen einer moderaten oder schweren Neuropathie auf", betonte Schnell. Bei etwa zwei Drittel aller Untersuchten, bei denen sich ein Neuropathie-Verdacht ergab, lag anamnestisch keine Neuropathie-Diagnose vor (61,5\% bei Typ-2-Diabetikern, 35,7\% bei Typ-1-Diabetikern und $79,1 \%$ bei Untersuchten ohne bekannten Diabetes). Insgesamt stieg der Anteil von NeuropathieVerdachtsfällen mit zunehmendem Lebensalter deutlich. Die Informationstour (www.hoerensieaufihrefuesse.de) ist bis November 2015 wieder in verschiedenen deutschen Städten unterwegs.

(ggi)

Pressekonferenz „Diabetes! Hören Sie auf Ihre Füße? Neues von der Aufklärungsinitiative zur diabetischen Neuropathie", 50. Jahrestagung der Deutschen Diabetes Gesellschaft (DDG); Berlin, 13.05.2015 (Veranstalter: Wörwag Pharma und Deutsche Diabetes-Stiftung (DDS)) 